\title{
Existence of Positive Solution to Schrödinger-type Semipositone Problems with Mixed Nonlinear Boundary Conditions
}

\author{
Eunkyung Ko, Eun Kyoung Lee* and Inbo Sim
}

\begin{abstract}
We studied the existence of a positive solution to Schrödinger-type semipositone problems with mixed nonlinear boundary conditions. By considering the cases when the reaction term with a parameter satisfies a superlinear and a sublinear growth condition at infinity, we established the existence of a positive solution for the large and small values of the parameter, respectively. The proofs are mainly based on the sub- and supersolution method for the sublinear case and the mountain pass lemma with $C^{1, \alpha}(\bar{\Omega})$-regularity for the superlinear case.
\end{abstract}

\section{Introduction and main results}

Owing to its suitability to describe a wide range of physical phenomena, the semilinear equation

$$
\begin{cases}-\Delta u=\lambda f(u), & x \in \Omega, \\ u=0, & x \in \partial \Omega\end{cases}
$$

related to reaction-diffusion models, has been widely and intensively studied for a long time. In (1.1) $\Delta u=\operatorname{div}(\nabla u), \Omega$ is a bounded domain in $\mathbb{R}^{N}, \partial \Omega$ is a smooth boundary of $\Omega, \lambda$ is a positive real parameter, and $f:[0, \infty) \rightarrow \mathbb{R}$ is a function. The existence of solutions of (1.1) according to the nonlinear behavior of $f$ has been of particular interest. Several researchers have investigated problems with nonnegative $f$ functions, while studies on models with sign-changing $f$ functions, specifically when $f(0)<0$, have been relatively rare. This case, known as the semipositone problem, has been extensively studied in the literature. Lions [31] reported that the study of positive solutions to semipositone problems is more challenging than the case of $f(0) \geq 0$.

Since then, several researchers have employed different conditions for (1.1). In one of these studies, a nonlinear boundary condition was applied instead of a Dirichlet boundary Received April 9, 2020; Accepted July 26, 2020.

Communicated by Eiji Yanagida.

2010 Mathematics Subject Classification. 35J66, 35J20.

Key words and phrases. existence, positive solution, semipositone problems, nonlinear boundary conditions, Schrödinger equations.

*Corresponding author. 
condition

$$
\begin{cases}-\Delta u=\lambda f(u), & x \in \Omega, \\ \frac{\partial u}{\partial \eta}+g(u) u=0, & x \in \partial \Omega,\end{cases}
$$

where $\frac{\partial}{\partial \eta}$ is an outward normal derivative and $g:[0, \infty) \rightarrow(0, \infty)$ is a function. The nonlinear boundary condition in $(1.2)$, naturally arises in several applications, for example, in thermal explosion models [23, 27], convection-diffusion systems, corrosion/oxidation models, and metal-insulator or metal-oxide semiconductor systems $3,6,15,18,26,34,37$.

Recently, Butler et al. [8] studied positive radial solutions to the boundary value problem on the exterior of a ball,

$$
\begin{cases}-\Delta u=\lambda K(|x|) f(u), & x \in \Omega, \\ \frac{\partial u}{\partial \eta}+g(u) u=0, & |x|=r_{0}, \\ u=0, & |x| \rightarrow \infty,\end{cases}
$$

where $\Omega=\left\{x \in \mathbb{R}^{N}: N>2,|x|>r_{0}\right.$ with $\left.r_{0}>0\right\}, K:\left(r_{0}, \infty\right) \rightarrow(0, \infty)$ is a continuous function such that $\lim _{r \rightarrow \infty} K(r)=0$ and $g$ is continuous. They transformed $(1.3)$ by the change of variable $r=|x|, t=\left(\frac{r}{r_{0}}\right)^{2-N}$, into a one-dimensional problem with a mixed nonlinear boundary condition

$$
\left\{\begin{array}{l}
-u^{\prime \prime}(t)=\lambda h(t) f(u(t)), \quad t \in(0,1), \\
\frac{(N-2)}{r_{0}} u^{\prime}(1)+g(u(1)) u(1)=0 \\
u(0)=0
\end{array}\right.
$$

where $h(t)=\frac{r_{0}^{2}}{(2-N)^{2}} t^{\frac{-2(N-2)}{N-2}} K\left(r_{0} t^{\frac{1}{2-N}}\right)$.

For simplicity, it is natural to consider an autonomous nonlinear partial differential equation (PDE) problem instead of the ordinary differential equation (ODE) above. Thus, in this paper, we are focusing on the existence of a positive solution of

$$
\begin{cases}-\Delta u+V(x) u=\lambda f(u), & x \in \Omega=\Omega_{1} \backslash \bar{\Omega}_{2}, \\ \frac{\partial u}{\partial \eta}+g(u) u=0, & x \in \partial \Omega_{1}, \\ u=0, & x \in \partial \Omega_{2},\end{cases}
$$

which allows $V \equiv 0$ (therefore, the Laplacian is its special case). In (1.4), $\Omega_{1}$ and $\Omega_{2}$ are subsets of $\Omega \subset \mathbb{R}^{N}$ with $\bar{\Omega}_{2} \subsetneq \Omega_{1}$, which are nonempty bounded domains in $\mathbb{R}^{N}, N>2$, $\partial \Omega_{1}$ is a smooth boundary of $\Omega_{1}$ with outward normal $\eta, \partial \Omega_{2}$ is a smooth boundary of $\Omega_{2}$ and $\lambda$ is a positive real parameter. In addition, $\partial \Omega=\partial \Omega_{1} \cup \partial \Omega_{2}$. Throughout this paper, we assume that 
(H) $V \in L^{\infty}(\Omega), f \in C^{1}([0, \infty)), g \in C^{\alpha}([0, \infty),(0, \infty))$ for some $0<\alpha<1$.

The first equation in (1.4) is derived based on the nonlinear Schrödinger equation, which is detailed in the seminal paper [36. Nonlinear Schrödinger equations have been intensively studied to demonstrate the existence of solutions that act according to $V$ on the whole space $\mathbb{R}^{N}$ (see $[5,24,32$ or references therein) or on bounded domains with linear boundary conditions under the assumption $f(0) \geq 0$ [19]. A numerical solution was treated in 28] for linear Schrödinger equations with a nonlinear boundary condition.

This paper presents the first ever investigation of the existence of a positive solution of (1.4) that includes the Laplacian as a special case under the simultaneous assumptions of $f(0)<0$ and a mixed nonlinear boundary condition when sufficient conditions on $V$, $f$, and $g$ are imposed.

In this study, we are interested in two different growth conditions on $f$ :

$\left(\mathrm{f}_{0}\right) \lim _{s \rightarrow \infty} \frac{f(s)}{s}=0$ (sublinear),

$\left(\mathrm{f}_{\infty}\right) \lim _{s \rightarrow \infty} \frac{f(s)}{s}=\infty$ (superlinear).

For the case $\left(f_{0}\right)$, sub- and supersolution methods have widely been used to obtain a positive solution for Laplacian semipositone problems, that is, the case of $V \equiv 0$ with Dirichlet boundary conditions on bounded domains (see $[2,4,10,16]$ ). Recently, such a method was applied to a Laplacian (not semipositone) problem with a nonlinear boundary condition 23 . We further assume that

$\left(\mathrm{F}_{1}\right) f(s) \rightarrow \infty$ as $s \rightarrow \infty$,

$\left(\mathrm{G}_{1}\right)$ there exists $M>0$ such that $g(s) \geq M$ for $s \geq 0$,

$\left(\mathrm{V}_{1}\right)$ there exists $c_{V}>0$ such that $V(x) \geq-c_{V}>-\frac{1}{\|e\|_{\infty}}$ for $x \in \Omega$, when $e$ is the positive solution of

$$
\begin{cases}-\Delta e=1 & \text { in } \Omega, \\ \frac{\partial e}{\partial \eta}+M e=0 & \text { on } \partial \Omega .\end{cases}
$$

Then, we establish our main result for the case when $f$ is sublinear near infinity.

Theorem 1.1. Let us assume $(\mathrm{H}),\left(\mathrm{f}_{0}\right),\left(\mathrm{F}_{1}\right),\left(\mathrm{G}_{1}\right)$, and $\left(\mathrm{V}_{1}\right)$. Then, for a sufficiently large $\lambda$, the problem (1.4) has a positive solution $u_{\lambda} \in C^{2}(\Omega) \cap C^{1, \alpha}(\bar{\Omega})$ for some $\alpha \in(0,1)$.

For the case $\left(f_{\infty}\right)$, the scaling method combined with the degree theory 13 and the mountain pass lemma with $C^{1, \alpha}(\bar{\Omega})$-regularity 9,35 are frequently used to demonstrate the existence of a positive solution for semipositone Laplacian (or $p$-Laplacian) problems with Dirichlet boundary conditions. Here, more precise assumptions are given for this case. First, to introduce a variational structure, we extend $f$ from $[0, \infty)$ to $\mathbb{R}$ such that $f \in C^{1}(\mathbb{R}, \mathbb{R})$ and assume that 
$\left(\mathrm{f}_{1}\right)$ there exist $A>0, B>0$ and $q \in\left(1, \frac{N+2}{N-2}\right)$ such that

$$
\begin{cases}A\left(u^{q}-1\right) \leq f(u) \leq B\left(u^{q}+1\right) & \text { for } u>0 \\ f(u)=0 & \text { for } u \leq-1 .\end{cases}
$$

We also extend $g$ from $[0, \infty)$ to $\mathbb{R}$ such that $g \in C^{\alpha}(\mathbb{R},(0, \infty))$ for some $\alpha \in(0,1)$ and assume that

$\left(\mathrm{g}_{1}\right)$ there exist $0<m_{g}<M_{g}<\infty$ such that $m_{g} \leq g(s) \leq M_{g}$ for all $s \in \mathbb{R}$, and $V$ satisfies

$\left(\mathrm{v}_{1}\right) V(x) \geq 0$ a.e. in $\Omega$.

We further assume Ambrosetti-Rabinowitz-type conditions such that

$\left(\mathrm{f}_{2}\right)$ there exist $\theta>\frac{2 M_{g}}{m_{g}}$ and $C \in \mathbb{R}$ such that

$$
u f(u) \geq \theta F(u)+C
$$

where $F(u)=\int_{0}^{u} f(s) d s$.

Remark 1.2. The condition (1.5) implies that $f$ satisfies the Ambrosetti-Rabinowitz condition as $\theta>\frac{2 M_{g}}{m_{g}}>2$.

Here, we establish our main result for the case when $f$ is superlinear near infinity.

Theorem 1.3. Let us assume $(\mathrm{H}),\left(\mathrm{f}_{1}\right),\left(\mathrm{f}_{2}\right),\left(\mathrm{g}_{1}\right)$, and $\left(\mathrm{v}_{1}\right)$. There exists $\lambda^{*}>0$ such that if $\lambda \in\left(0, \lambda^{*}\right)$, then the equation (1.4) has a positive solution $u_{\lambda} \in C^{2}(\Omega) \cap C^{1, \beta}(\bar{\Omega})$ for some $\beta \in(0,1)$.

Remark 1.4. (i) We emphasize that our two main results remain valid even for the case $V \equiv 0$. In this case, the main problem in Theorem 1.3 is that the coercive term, such as $V(x) u$, disappears. To overcome this, we observe that the coercivity induced by the boundary condition from the assumption $g(u) u$ is strictly positive. This motivates the definition of an equivalent norm in $H^{1}(\Omega)$ defined in (3.1), with respect to which the energy functional corresponding to 1.4 can be analyzed.

(ii) The condition on $g$ is restrictive. For the more general case for $g$, we refer to [28], where it is treated by a linear Schrödinger equation.

This paper is organized as follows: In Section 2, we establish the method of obtaining sub- and supersolutions for (1.4) and provide a proof of Theorem 1.1. In Section 3 , we present a proof of Theorem 1.3 by using the mountain pass argument. 


\section{Sublinear semipositone}

In this section, we establish the method of obtaining sub- and supersolutions for (1.4) and provide a proof of Theorem 1.1 .

\subsection{Method of sub- and supersolutions}

A subsolution of $(1.4)$ is defined as a function $\psi: \bar{\Omega} \rightarrow \mathbb{R}$ satisfying

$$
\begin{cases}-\Delta \psi+V(x) \psi \leq \lambda f(\psi), & x \in \Omega, \\ \frac{\partial \psi}{\partial \eta}+g(\psi) \psi \leq 0, & x \in \partial \Omega_{1}, \\ \psi \leq 0, & x \in \partial \Omega_{2}\end{cases}
$$

while a supersolution of $(1.4)$ is defined as a function $\phi: \bar{\Omega} \rightarrow \mathbb{R}$ satisfying

$$
\begin{cases}-\Delta \phi+V(x) \phi \geq \lambda f(\phi), & x \in \Omega, \\ \frac{\partial \phi}{\partial \eta}+g(\phi) \phi \geq 0, & x \in \partial \Omega_{1} \\ \phi \geq 0, & x \in \partial \Omega_{2} .\end{cases}
$$

To prove our second result, the following lemma is used.

Lemma 2.1. If a subsolution $\psi$ and a supersolution $\phi$ of 1.4 exist such that $\psi \leq \phi$ on $\bar{\Omega}$, then (1.4) has at least one solution $u \in C^{2}(\Omega) \cap C^{1, \alpha}(\bar{\Omega})$ satisfying $\psi \leq u \leq \phi$ on $\bar{\Omega}$.

Proof. By applying the results in [1] and [25] to (1.4), it can be shown that, under the ordering assumptions of this lemma, there exists $u \in W^{2, p}(\Omega)$ for $p>N$. Thus, based on the Sobolev embedding theorem $u \in C^{1, \alpha}(\bar{\Omega})$ (see $[17$, Theorem 6, p. 270]). This implies that $f(u) \in C^{1}(\bar{\Omega})$ and $g(u) u \in C^{\alpha}(\bar{\Omega})$. Consequently, according to 22, Theorem 6.25] and 30 , Theorem 2], $u \in C^{2}(\Omega) \cap C^{1, \alpha}(\bar{\Omega})$ is a classical solution of 1.4 .

Lemma 2.2. Let us assume $\left(\mathrm{V}_{1}\right)$. Then, the following problem

$$
\begin{cases}-\Delta w+V(x) w=1 & \text { in } \Omega, \\ \frac{\partial w}{\partial \eta}+M w=0 & \text { on } \partial \Omega\end{cases}
$$

has a solution $w$ such that $w(x)>0$ for $x \in \bar{\Omega}$ and $\frac{\partial w}{\partial \eta}<0$ on $\partial \Omega$.

Proof. For $K>0$ such that $\frac{1}{K} \leq 1-c_{V}\|e\|_{\infty}$, let $z=K e$, then

$$
\begin{aligned}
-\Delta z+V(x) z & =K(-\Delta e+V(x) e) \\
& =K(1+V(x) e) \geq K\left(1-c_{V} e\right) \geq K\left(1-c_{V}\|e\|_{\infty}\right) \geq 1
\end{aligned}
$$


and $\frac{\partial z}{\partial \eta}+M z=0$ on $\partial \Omega$. Thus, $z$ is a supersolution of 2.1 and $z(x)>0$ for $x \in \bar{\Omega}$. As zero is the trivial subsolution of (2.1) but not a solution of (2.1), according to the sub-supersolution theorem, there exists a solution $w$ of $(2.1)$ such that $0 \leq w(x) \leq z(x)$. Here, we claim that $w(x)>0$ for $x \in \Omega$ and $\frac{\partial w}{\partial \eta}<0$ on $\partial \Omega$. Otherwise, there exists $x_{0} \in \Omega$ such that $w\left(x_{0}\right)=0$. Then, we find that $1=-\Delta w\left(x_{0}\right)+V\left(x_{0}\right) w\left(x_{0}\right) \leq 0$ as $\|V\|<\infty$, which is a contradiction. Hence, $w>0$ in $\Omega$, and using the Hopf maximum principle, we obtain $\frac{\partial w}{\partial \eta}<0$ on $\partial \Omega$. Finally, we conclude that $w>0$ for all $x \in \bar{\Omega}$ because $w$ satisfies the boundary condition of 2.1 .

Let $\lambda_{1}$ and $\phi_{1}$ be the first eigenvalue and the corresponding eigenfunction of

$$
\begin{cases}-\Delta \phi+V(x) \phi=\lambda \phi & \text { in } \Omega, \\ \phi=0 & \text { on } \partial \Omega .\end{cases}
$$

Then, $\phi_{1}(x)>0$ for $x \in \Omega$ and $\frac{\partial \phi_{1}}{\partial \eta}<0$ on $\partial \Omega 14,20,33$.

\subsection{Proof of Theorem 1.1}

First, we construct a subsolution. Since $\phi_{1}>0$ in $\Omega$ and $\frac{\partial \phi_{1}}{\partial \eta}<0$ on $\partial \Omega$, there exist $\delta>0$ and $m>0$ such that

$$
2\left|\nabla \phi_{1}\right|^{2}-\left(2 \lambda_{1}+c_{V}\right) \phi_{1}^{2} \geq m \quad \text { in }\{x \in \Omega \mid d(x, \partial \Omega)<\delta\}
$$

Define $\Omega_{\delta}:=\{x \in \Omega \mid d(x, \partial \Omega)<\delta\}$. Then, there exists $\mu>0$ such that

$$
\phi_{1}^{2} \geq \mu \quad \text { in } \Omega \backslash \Omega_{\delta}
$$

Let $\underline{f}=\min _{s \in[0, \infty)} f(s)<0$. From $\left(\mathrm{F}_{1}\right)$, we find that there exists $L>0$ such that

$$
\frac{(-\underline{f})}{m}\left(\lambda_{1}+c_{V}\right)<f(s) \text { for } s>L
$$

and such that

$$
\frac{\lambda(-\underline{f})}{m} \mu>L \quad \text { for sufficiently large } \lambda \gg 1 .
$$

Let us denote $\rho:=\frac{\lambda(-\underline{f})}{m}$ and define $\psi=\rho \phi_{1}^{2}$. We estimate $-\Delta \psi+V(x) \psi$ in $\Omega_{\delta}$ as

$$
\begin{aligned}
-\Delta \psi+V(x) \psi & =-2 \rho\left|\nabla \phi_{1}\right|^{2}+2 \rho\left(\lambda_{1}-V(x)\right) \phi_{1}^{2}+\rho V(x) \phi_{1}^{2} \\
& =-2 \rho\left|\nabla \phi_{1}\right|^{2}+2 \rho \lambda_{1} \phi_{1}^{2}-\rho V(x) \phi_{1}^{2} \\
& \leq-\rho\left(2\left|\nabla \phi_{1}\right|^{2}-\left(2 \lambda_{1}+c_{V}\right) \phi_{1}^{2}\right) \\
& \leq-\rho m=\lambda \underline{f} \leq \lambda f(\psi) .
\end{aligned}
$$


We also have in $\Omega \backslash \Omega_{\delta}$, from 2.2 and $(2.3)$,

$$
\begin{aligned}
-\Delta \psi+V(x) \psi & \leq-\rho\left(2\left|\nabla \phi_{1}\right|^{2}-\left(2 \lambda_{1}+c_{V}\right) \phi_{1}^{2}\right) \leq \frac{\lambda(-\underline{f})}{m}\left(2 \lambda_{1}+c_{V}\right) \phi_{1}^{2} \\
& \leq \frac{\lambda(-\underline{f})}{m}\left(2 \lambda_{1}+c_{V}\right) \leq \lambda f\left(\frac{\lambda(-\underline{f})}{m} \phi_{1}^{2}\right)=\lambda f(\psi)
\end{aligned}
$$

as $\mu \leq \phi_{1}^{2} \leq 1$. Since $\psi=0$ and $\frac{\partial \psi}{\partial \eta}+g(\psi) \psi=0$ on $\partial \Omega, \psi$ is a subsolution of (1.4) for $\lambda \gg 1$.

Next, we construct a supersolution. Let us define $\bar{f}(s):=\max _{t \leq s} f(t)$. Then, $f(s) \leq$ $\bar{f}(s), \bar{f}$ is monotone increasing and $\lim _{s \rightarrow \infty} \frac{\bar{f}(s)}{s}=0$. This implies that there exists $M_{\lambda} \gg 1$ such that $M_{\lambda} w \geq \psi$ and satisfies

$$
\frac{\bar{f}\left(M_{\lambda}\|w\|_{\infty}\right)}{M_{\lambda}\|w\|_{\infty}} \leq \frac{1}{\lambda\|w\|_{\infty}} .
$$

Let $\zeta=M_{\lambda} w$. Then, using (2.4) and the definition of $\bar{f}$, we can write

$$
\begin{aligned}
-\Delta \zeta+V(x) \zeta & =M_{\lambda}(-\Delta w+V(x) w)=M_{\lambda} \\
& \geq \lambda \bar{f}\left(M_{\lambda}\|w\|_{\infty}\right) \geq \lambda \bar{f}\left(M_{\lambda} w\right) \geq \lambda f\left(M_{\lambda} w\right)=\lambda f(\zeta) .
\end{aligned}
$$

Moreover, on $\partial \Omega, \zeta>0$ and

$$
\frac{\partial \zeta}{\partial \eta}+g(\zeta) \zeta=M_{\lambda}\left(\frac{\partial w}{\partial \eta}+g\left(M_{\lambda} w\right) w\right) \geq M_{\lambda}\left(\frac{\partial w}{\partial \eta}+M w\right)=0 .
$$

By considering a sufficiently large $M_{\lambda}$ such that $M_{\lambda} w \geq \rho \phi_{1}^{2}$ in $\Omega, \zeta$ becomes a supersolution satisfying $\psi \leq \zeta$. Thus, the proof is completed.

\section{Superlinear semipositone}

In this section, we present a proof of Theorem 1.3 by using the mountain pass argument.

\subsection{Preliminary}

Let $H^{1}(\Omega)=\left\{u: u \in L^{2}(\Omega), \nabla u \in\left(L^{2}(\Omega)\right)^{N}\right\}$ be a standard Sobolev space with the norm $\|u\|_{H^{1}(\Omega)}^{2}=\int_{\Omega}\left(|\nabla u|^{2}+|u|^{2}\right)$. We define

$$
H:=\left\{u \in H^{1}(\Omega): u=0 \text { on } \partial \Omega_{2}\right\}
$$

and the inner product

$$
(u, v):=\int_{\Omega} \nabla u \cdot \nabla v d x+m_{g} \int_{\partial \Omega_{1}} u v d \sigma, \quad \forall u, v \in H
$$


where $d \sigma$ denotes the surface measure on $\partial \Omega_{1}$ and $m_{g}>0$ is defined in $\left(\mathrm{g}_{1}\right)$. Then, $H$ is a Hilbert space with an associated norm

$$
\|u\|_{H}^{2}:=\int_{\Omega}|\nabla u|^{2} d x+m_{g} \int_{\partial \Omega_{1}} u^{2} d \sigma, \quad \forall u \in H .
$$

Owing to the imbedding provided by Cherrier [11,12, 29], there exists $c_{I}, c_{I I}>0$ such that

$$
c_{I}\|u\|_{H^{1}(\Omega)} \leq\|u\|_{H} \leq c_{I I}\|u\|_{H^{1}(\Omega)}, \quad \forall u \in H^{1}(\Omega) .
$$

Thus, the space $H$ is complete with respect to the norm $\|\cdot\|_{H}$. A weak solution of (1.4) is defined as $u \in H$ satisfying

$$
\int_{\Omega}(\nabla u \cdot \nabla \psi+V(x) u \psi) d x-\lambda \int_{\Omega} f(u) \psi d x+\int_{\partial \Omega_{1}} g(u) u \psi d \sigma=0 \quad \text { for all } \psi \in H .
$$

We define the energy functional $E_{\lambda}$ associated with the problem 1.4 as

$$
E_{\lambda}(u)=\frac{1}{2} \int_{\Omega}\left(|\nabla u|^{2}+V(x) u^{2}\right) d x-\lambda \int_{\Omega} F(u) d x+\int_{\partial \Omega_{1}} G(u) d \sigma, \quad u \in H,
$$

where $G(t):=\int_{0}^{t} g(s) s d s$. It is well-known that $E_{\lambda}$ is a $C^{1}$ functional and the critical points of $E_{\lambda}$ are the weak solutions of $(1.4)$. We denote the norm in the $L^{p}(\Omega)$ space by $\|\cdot\|_{p}$ and the norm in the Sobolev space $W^{1, p}(\Omega)$ by $\|\cdot\|_{1, p}$.

\subsection{Existence of a solution of mountain pass type}

In this subsection, we prove that $E_{\lambda}$ satisfies the mountain pass geometry in the first two lemmas and equation (1.4) has a solution $u_{\lambda} \in H_{D}^{1}(\Omega)$ using the mountain pass theorem in the last lemma. Before stating the lemmas, we note that, from the condition $\left(\mathrm{f}_{1}\right)$, there exist $A_{1}>0, B_{1}>0$ such that

$$
F(u) \leq B_{1}\left(|u|^{q+1}+1\right) \quad \text { for all } u \in \mathbb{R}
$$

and

$$
F(u) \geq A_{1}\left(|u|^{q+1}-1\right) \quad \text { for all } u \geq 0
$$

For simplicity, we denote $r=\frac{1}{q-1}$ and let $\psi \in H$ be a positive function with $\|\psi\|_{H}=1$. Let us define a constant

$$
\mu=\left(\frac{M_{g}+m_{g}\|V\|_{\infty}\|\psi\|_{2}^{2}}{A_{1} m_{g}\|\psi\|_{q+1}^{q+1}}\right)^{r}
$$

which is used in the following lemma. 
Lemma 3.1. There exists $\lambda_{1}>0$ such that if $\lambda \in\left(0, \lambda_{1}\right)$, then $E_{\lambda}\left(\mu \lambda^{-r} \psi\right) \leq 0$.

Proof. Let $s=\mu \lambda^{-r}$. Using (3.4), we obtain

$$
\begin{aligned}
E_{\lambda}(s \psi) & =\frac{s^{2}}{2} \int_{\Omega}\left(|\nabla \psi|^{2}+V(x) \psi^{2}\right) d x-\lambda \int_{\Omega} F(s \psi) d x+\int_{\partial \Omega_{1}} G(s \psi) d \sigma \\
& \leq \frac{s^{2}}{2} \int_{\Omega}\left(|\nabla \psi|^{2}+V(x) \psi^{2}\right) d x-\lambda A_{1} \int_{\Omega}\left(s^{q+1}|\psi|^{q+1}-1\right) d x+\frac{M_{g}}{2} \int_{\partial \Omega_{1}} s^{2} \psi^{2} d \sigma \\
& \leq \frac{s^{2}}{2} \frac{M_{g}}{m_{g}}\|\psi\|_{H}^{2}+\frac{s^{2}}{2}\|V\|_{\infty}\|\psi\|_{2}^{2}-\lambda A_{1} s^{q+1}\|\psi\|_{q+1}^{q+1}+\lambda A_{1}|\Omega| .
\end{aligned}
$$

By substituting (3.5) into (3.6), we find

$$
\begin{aligned}
E_{\lambda}(s \psi) & \leq \frac{\mu^{2} \lambda^{-2 r}}{2} \frac{M_{g}}{m_{g}}+\frac{\mu^{2} \lambda^{-2 r}}{2}\|V\|_{\infty}\|\psi\|_{2}^{2}-\lambda A_{1} \mu^{q+1} \lambda^{-r(q+1)}\|\psi\|_{q+1}^{q+1}+\lambda A_{1}|\Omega| \\
& =\mu^{2} \lambda^{-2 r}\left[\frac{1}{2}\left(\frac{M_{g}}{m_{g}}+\|V\|_{\infty}\|\psi\|_{2}^{2}\right)-A_{1} \mu^{q-1}\|\psi\|_{q+1}^{q+1}\right]+\lambda A_{1}|\Omega| \\
& =-\frac{\mu^{2} \lambda^{-2 r}}{2}\left(\frac{M_{g}}{m_{g}}+\|V\|_{\infty}\|\psi\|_{2}^{2}\right)+\lambda A_{1}|\Omega| .
\end{aligned}
$$

By taking $\lambda_{1}<\min \left\{1,\left(\frac{\mu^{2}\left(M_{g}+m_{g}\|V\|_{\infty}\|\psi\|_{2}^{2}\right)}{2 A_{1} m_{g}|\Omega|}\right)^{\frac{1}{1+2 r}}\right\}$, the lemma is proven.

Lemma 3.2. There exist $\tau \in(0, \mu)$ and $\lambda_{2} \in(0,1)$ such that, if $\|u\|_{H}=\tau \lambda^{-r}$, then $E_{\lambda}(u) \geq \frac{1}{8}\left(\tau \lambda^{-r}\right)^{2}$ for all $\lambda \in\left(0, \lambda_{2}\right)$.

Proof. First, we notice that there exists $K_{1}>0$ such that $\|u\|_{q+1} \leq K_{1}\|u\|_{H}$ for $u \in H$ according to the Sobolev embedding theorem and (3.2). Let

$$
\tau<\min \left\{\left(4 B_{1} K_{1}^{q+1}\right)^{-r}, \mu\right\} .
$$

If $\|u\|_{H}=\tau \lambda^{-r}$, then, according to (3.4) and because $V(x) \geq 0$ a.e. in $\Omega$,

$$
\begin{aligned}
E_{\lambda}(u) & =\frac{1}{2} \int_{\Omega}\left(|\nabla u|^{2}+V(x) u^{2}\right) d x-\lambda \int_{\Omega} F(u) d x+\int_{\partial \Omega_{1}} G(u) d \sigma \\
& \geq \frac{1}{2} \int_{\Omega}|\nabla u|^{2} d x-\lambda \int_{\Omega} B_{1}\left(|u|^{q+1}+1\right) d x+\frac{m_{g}}{2} \int_{\partial \Omega_{1}} u^{2} d \sigma \\
& \geq \frac{1}{2}\|u\|_{H}^{2}-\lambda B_{1}\|u\|_{q+1}^{q+1}-\lambda B_{1}|\Omega| \\
& \geq \frac{1}{2}\|u\|_{H}^{2}-\lambda B_{1} K_{1}^{q+1}\|u\|_{H}^{q+1}-\lambda B_{1}|\Omega| \\
& =\lambda^{-2 r}\left[\frac{1}{2} \tau^{2}-B_{1} K_{1}^{q+1} \tau^{q+1}-\lambda^{1+2 r} B_{1}|\Omega|\right] \\
& \geq \lambda^{-2 r}\left[\frac{1}{4} \tau^{2}-\lambda^{1+2 r} B_{1}|\Omega|\right]
\end{aligned}
$$


where (3.7) is used at the last inequality. Next, we consider $\lambda>0$ such that $\lambda<$ $\left(\frac{\tau^{2}}{8 B_{1}|\Omega|}\right)^{\frac{1}{1+2 r}}:=\lambda_{2}$. Then, $E_{\lambda}(u) \geq \frac{1}{8}\left(\tau \lambda^{-r}\right)^{2}$ if $\|u\|_{H}=\tau \lambda^{-r}$.

Lemma 3.3. Let $\lambda_{3}=\min \left\{\lambda_{1}, \lambda_{2}, 1\right\}$. There exists $c_{1}>0$ such that for each $\lambda \in\left(0, \lambda_{3}\right)$, the functional $E_{\lambda}$ has a mountain pass type critical point $u_{\lambda}$ satisfying $E_{\lambda}\left(u_{\lambda}\right) \leq c_{1} \lambda^{-2 r}$.

Proof. First, we show that $E_{\lambda}$ satisfies the Palais-Smale condition. We assume that $\left\{u_{n}\right\}$ is a sequence in $H$ such that $\left\{E_{\lambda}\left(u_{n}\right)\right\}$ is bounded and $\lim _{n \rightarrow \infty}\left\|E_{\lambda}^{\prime}\left(u_{n}\right)\right\|_{H^{*}}=0$. By setting $\rho>0$ as a constant such that $\left|E_{\lambda}\left(u_{n}\right)\right| \leq \rho$ for all $n=1,2, \ldots$, we have

$$
\frac{1}{2} \int_{\Omega}\left(\left|\nabla u_{n}\right|^{2}+V(x) u_{n}^{2}\right) d x-\lambda \int_{\Omega} F\left(u_{n}\right) d x+\int_{\partial \Omega_{1}} G\left(u_{n}\right) d \sigma \leq \rho
$$

and

$$
\begin{aligned}
& \left|\int_{\Omega}\left(\nabla u_{n} \cdot \nabla \psi+V(x) u_{n} \psi\right) d x-\lambda \int_{\Omega} f\left(u_{n}\right) \psi d x-\int_{\partial \Omega_{1}} g\left(u_{n}\right) u_{n} \psi d \sigma\right| \\
= & o(1)\|\psi\|_{H}, \quad \forall \psi \in H .
\end{aligned}
$$

Notably, from (3.8), we find that due to the condition $\left(\mathrm{f}_{2}\right)$,

$$
\begin{aligned}
\frac{1}{2}\left\|u_{n}\right\|_{H}^{2}+\frac{1}{2} \int_{\Omega} V(x) u_{n}^{2} d x & \leq \rho+\lambda \int_{\Omega} F\left(u_{n}\right) d x \\
& \leq \rho+\frac{\lambda}{\theta} \int_{\Omega}\left(f\left(u_{n}\right) u_{n}-C\right) d x
\end{aligned}
$$

Then, substituting $u_{n}$ for $\psi$ into $(3.9)$, we obtain

$$
\begin{aligned}
\lambda \int_{\Omega} f\left(u_{n}\right) u_{n} d x & \leq \int_{\Omega}\left(\left|\nabla u_{n}\right|^{2}+V(x) u_{n}^{2}\right) d x+M_{g} \int_{\partial \Omega_{1}} u_{n}^{2} d \sigma+o(1)\left\|u_{n}\right\|_{H} \\
& \leq \frac{M_{g}}{m_{g}}\left\|u_{n}\right\|_{H}^{2}+\int_{\Omega} V(x) u_{n}^{2} d x+o(1)\left\|u_{n}\right\|_{H} .
\end{aligned}
$$

By combining (3.10) and (3.11), as $\frac{1}{\theta}<\frac{M_{g}}{m_{g} \theta}<\frac{1}{2}$, it follows that $\sup _{n}\left\|u_{n}\right\|_{H} \leq c<\infty$ for some $c>0$, which implies that $\left\{u_{n}\right\}$ is bounded in $H$. Therefore, there exists $u_{\lambda} \in H$ such that

$$
u_{n} \rightarrow u_{\lambda} \text { in } H
$$

It should be noted that, due to the compact embedding $H^{1}(\Omega) \hookrightarrow L^{p}(\Omega)$ for all $1 \leq p<$ $\frac{2 N}{N-2}=: 2^{*}$, there exists a subsequence $\left\{u_{n_{k}}\right\}$, denoted by $\left\{u_{n}\right\}$, such that $u_{n} \rightarrow u_{\lambda}$ in $L^{p}(\Omega)$ for all $1 \leq p<2^{*}$. Thus, we can write

$$
\int_{\Omega} V(x) u_{n} \psi d x \rightarrow \int_{\Omega} V(x) u_{\lambda} \psi d x, \quad \int_{\Omega} f\left(u_{n}\right) \psi d x \rightarrow \int_{\Omega} f\left(u_{\lambda}\right) \psi d x, \quad \forall \psi \in H,
$$


and

$$
\int_{\Omega} V(x) u_{n}^{2} d x \rightarrow \int_{\Omega} V(x) u_{\lambda}^{2} d x, \quad \int_{\Omega} f\left(u_{n}\right) u_{n} d x \rightarrow \int_{\Omega} f\left(u_{\lambda}\right) u_{\lambda} d x
$$

as $n \rightarrow \infty$. Due to the compactness of trace embedding $H^{1}(\Omega) \hookrightarrow L^{2}(\partial \Omega)$,

$$
\begin{gathered}
\int_{\partial \Omega_{1}} g\left(u_{n}\right) u_{n} \psi d \sigma \rightarrow \int_{\partial \Omega_{1}} g\left(u_{\lambda}\right) u_{\lambda} \psi d \sigma, \quad \forall \psi \in H, \\
\int_{\partial \Omega_{1}} g\left(u_{n}\right) u_{n}^{2} d \sigma \rightarrow \int_{\partial \Omega_{1}} g\left(u_{\lambda}\right) u_{\lambda}^{2} d \sigma
\end{gathered}
$$

as $n \rightarrow \infty$. Using (3.8), 3.12), (3.13) and (3.15), we find that $u_{\lambda}$ is a weak solution of (1.4) and from (3.8), (3.14) and (3.16), it follows that $\int_{\Omega}\left|\nabla u_{n}\right|^{2} \rightarrow \int_{\Omega}\left|\nabla u_{\lambda}\right|^{2}$ as $n \rightarrow \infty$. This proves that $E_{\lambda}$ satisfies the Palais-Smale condition.

Next, using (3.6) we can write

$$
E_{\lambda}(s \psi) \leq \frac{s^{2}}{2} \frac{M_{g}}{m_{g}}\|\psi\|_{H}^{2}+\frac{s^{2}}{2}\|V\|_{\infty}\|\psi\|_{2}^{2}-\lambda A_{1} s^{q+1}\|\psi\|_{q+1}^{q+1}+\lambda A_{1}|\Omega| .
$$

Let us denote $H(s):=s^{2} D-s^{q+1} E+\lambda A_{1}|\Omega|$, where $D=\frac{1}{2}\left(\frac{M_{g}}{m_{g}}\|\psi\|_{H}^{2}+\|V\|_{\infty}\|\psi\|_{2}^{2}\right)$ and $E=A_{1}\|\psi\|_{q+1}^{q+1}$. Then, $H(s)$ reaches its maximum at the value of $s=\left(\frac{2 D}{(q+1) E}\right)^{r}$. Consequently, we obtain that

$$
\begin{aligned}
\max _{s \geq 0} E_{\lambda}(s \psi) & \leq \frac{2^{2 r} D^{1+2 r}(q-1)}{E^{2 r}(q+1)^{2 r+1}} \lambda^{-2 r}+\lambda\left|A_{1}\right||\Omega| \\
& =: \widetilde{c}_{1} \lambda^{-2 r}+\lambda\left|A_{1}\right||\Omega| \leq \widetilde{c}_{1} \lambda^{-2 r}+\lambda^{-2 r}\left|A_{1}\right||\Omega|=: c_{1} \lambda^{-2 r} .
\end{aligned}
$$

Therefore, according to the mountain pass theorem, there exists $u_{\lambda} \in H$ such that $E_{\lambda}^{\prime}\left(u_{\lambda}\right)=0$ and

$$
\frac{1}{8}\left(\tau \lambda^{-r}\right)^{2} \leq E_{\lambda}\left(u_{\lambda}\right) \leq c_{1} \lambda^{-2 r}
$$

\subsection{Positivity of solutions}

Lemma 3.4. Let $u_{\lambda} \in H$ be the solution in Lemma 3.3. Then $u_{\lambda} \in C^{1, \alpha}(\bar{\Omega})$ for some $\alpha \in(0,1)$.

Proof. Based on the same argument as that in Theorem 1.2 in [21], it can be shown that any solution of $(1.4)$ is bounded. Furthermore, we conclude that $u_{\lambda} \in C^{1, \alpha}(\bar{\Omega})$ using Theorem 2 in 30 .

Lemma 3.5. Let $u_{\lambda} \in H$ be a solution in Lemma 3.3. Then, there exists $M_{1}>0$ such that

$$
M_{1} \lambda^{-r} \leq\|u\|_{\infty}
$$


Proof. According to Lemma 3.2, $E_{\lambda}\left(u_{\lambda}\right) \geq \frac{1}{8}\left(\tau \lambda^{-r}\right)^{2}=: c_{1} \lambda^{-2 r}$. Considering that $F(s) \geq$ $\min F>-\infty$ and $f(s) s \leq B_{1}\left(|s|^{q+1}+|s|\right)$ for all $s \in \mathbb{R}$, we obtain

$$
\begin{aligned}
\lambda \int_{\Omega} f\left(u_{\lambda}\right) u_{\lambda} d x & =\int_{\Omega}\left(\left|\nabla u_{\lambda}\right|^{2}+V(x) u_{\lambda}^{2}\right) d x+\int_{\partial \Omega_{1}} g\left(u_{\lambda}\right) u_{\lambda}^{2} d \sigma \\
& =2 E_{\lambda}\left(u_{\lambda}\right)+2 \lambda \int_{\Omega} F\left(u_{\lambda}\right) d x-2 \int_{\partial \Omega_{1}} G\left(u_{\lambda}\right) d \sigma+\int_{\partial \Omega_{1}} g\left(u_{\lambda}\right) u_{\lambda}^{2} d \sigma \\
& \geq \frac{1}{4}\left(\tau \lambda^{-r}\right)^{2}+2 \lambda|\Omega| \min F-\left(M_{g}-m_{g}\right)\left\|u_{\lambda}\right\|_{L^{2}\left(\partial \Omega_{1}\right)}^{2}
\end{aligned}
$$

which implies that there exists $\widetilde{c}>0$ such that $\left(M_{g}-m_{g}\right)\left\|u_{\lambda}\right\|_{L^{2}\left(\partial \Omega_{1}\right)}^{2}+\lambda \int_{\Omega} f\left(u_{\lambda}\right) u_{\lambda} d x \geq$ $\widetilde{c} \lambda^{-2 r}$. Thus, $\lim _{\lambda \rightarrow 0}\left\|u_{\lambda}\right\|_{\infty}=+\infty$. Nevertheless, according to $\left(\mathrm{f}_{1}\right)$,

$$
\begin{aligned}
\lambda \int_{\Omega} f\left(u_{\lambda}\right) u_{\lambda} d x & \leq B_{1} \lambda \int_{\Omega}\left(\left|u_{\lambda}\right|^{q+1}+\left|u_{\lambda}\right|\right) d x \\
& \leq 2 B_{1} \lambda|\Omega|\left\|u_{\lambda}\right\|_{\infty}^{q+1}
\end{aligned}
$$

holds, based on the fact that $0<\lambda<1$. Thus, we obtain

$$
\begin{aligned}
\widetilde{c} \lambda^{-2 r} & \leq 2 B_{1}|\Omega| \lambda\left\|u_{\lambda}\right\|_{\infty}^{q+1}+\left(M_{g}-m_{g}\right)\left\|u_{\lambda}\right\|_{L^{2}\left(\partial \Omega_{1}\right)}^{2} \\
& \leq\left(2 B_{1}|\Omega| \lambda+\left(M_{g}-m_{g}\right)\left|\partial \Omega_{1}\right|\right)\left\|u_{\lambda}\right\|_{\infty}^{q+1} .
\end{aligned}
$$

Consequently, we find that

$$
\left\|u_{\lambda}\right\|_{\infty}^{q+1} \geq d_{1} \lambda^{-2 r-1} \quad \text { or } \quad\left\|u_{\lambda}\right\|_{\infty}^{q+1} \geq d_{2} \lambda^{-2 r}
$$

where $d_{1}=\frac{\widetilde{c}}{2}\left(2 B_{1}|\Omega|\right)^{-1}$ and $d_{2}=\frac{\widetilde{c}}{2}\left(\left(M_{g}-m_{g}\right)\left|\partial \Omega_{1}\right|\right)^{-1}$, which implies that

$$
\left\|u_{\lambda}\right\|_{\infty}^{q+1} \geq d_{1} \lambda^{-2 r-1} \quad \text { for sufficiently small } \lambda \text {. }
$$

By setting $M_{1}=\left(d_{1}\right)^{\frac{1}{q+1}}$, the proof is completed.

Lemma 3.6. Let $u_{\lambda} \in H$ be the same as that in Lemma 3.3. Then, there exists $c_{2}>0$ such that

$$
\|u\|_{H^{1}(\Omega)}^{2} \leq c_{2} \lambda^{-2 r}, \quad \forall \lambda \in\left(0, \lambda_{3}\right)
$$

Proof. As $V(x) \geq 0$, according to (3.3), we obtain

$$
\int_{\Omega}\left|\nabla u_{\lambda}\right|^{2} d x+\int_{\partial \Omega_{1}} g\left(u_{\lambda}\right) u_{\lambda}^{2} d \sigma \leq \lambda \int_{\Omega} f\left(u_{\lambda}\right) u_{\lambda} d x .
$$


Multiplying (3.17) by $\left(1-\frac{2}{\theta}\right)$ and using 1.5 , we find

$$
\begin{aligned}
& \left(1-\frac{2}{\theta}\right)\left[\int_{\Omega}\left|\nabla u_{\lambda}\right|^{2} d x+\int_{\partial \Omega_{1}} g\left(u_{\lambda}\right) u_{\lambda}^{2} d \sigma\right] \\
\leq & \lambda \int_{\Omega} f\left(u_{\lambda}\right) u_{\lambda} d x-2 \lambda \int_{\Omega} \frac{1}{\theta} f\left(u_{\lambda}\right) u_{\lambda} d x \\
\leq & \lambda \int_{\Omega} f\left(u_{\lambda}\right) u_{\lambda} d x-2 \lambda \int_{\Omega}\left[F\left(u_{\lambda}\right)+\frac{C}{\theta}\right] d x \\
\leq & 2 E\left(u_{\lambda}\right)-2 \int_{\partial \Omega_{1}} G\left(u_{\lambda}\right) d \sigma+\int_{\partial \Omega_{1}} g\left(u_{\lambda}\right) u_{\lambda}^{2} d \sigma+\frac{2 \lambda|C|}{\theta} .
\end{aligned}
$$

Based on condition (g), we obtain

$$
\left(1-\frac{2}{\theta}\right) \int_{\Omega}\left|\nabla u_{\lambda}\right|^{2} d x+\left(m_{g}-\frac{2 M_{g}}{\theta}\right) \int_{\partial \Omega_{1}} u_{\lambda}^{2} d \sigma \leq 2 c_{1} \lambda^{-2 r}+\frac{2 \lambda|C|}{\theta} \leq \bar{c} \lambda^{-2 r}
$$

for some $\bar{c}>0$, which implies that $\|u\|_{H^{1}(\Omega)}^{2} \leq c_{2} \lambda^{-2 r}$ with $c_{2}:=c_{I I}^{-1}\left(1-\frac{2}{\theta} \frac{M_{g}}{m_{g}}\right)^{-1} \bar{c}$.

Proof of Theorem 1.3. Let us suppose on the contrary that there exists a sequence $\left\{\lambda_{j}\right\}$, $0<\lambda_{j}<1$ converging to 0 such that the measure $m\left(\left\{x \in \Omega: u_{\lambda_{j}}(x) \leq 0\right\}\right)>0$, where $u_{\lambda_{j}}$ is a weak solution corresponding to $\lambda_{j}$. Let $w_{j}=\frac{u_{\lambda_{j}}}{\left\|u_{\lambda_{j}}\right\|_{\infty}}$. Then, we can write

$$
\begin{cases}-\Delta w_{j}+V(x) w_{j}=\lambda f\left(u_{\lambda_{j}}\right)\left\|u_{\lambda_{j}}\right\|_{\infty}^{-1}, & x \in \Omega, \\ \frac{\partial w_{j}}{\partial \eta}+g\left(u_{\lambda_{j}}\right) w_{j}=0, & x \in \partial \Omega_{1}, \\ u=0, & x \in \partial \Omega_{2} .\end{cases}
$$

According to Lemmas 3.5 and 3.6 , there exists a constant $c_{3}>0$ such that

$$
\left\|w_{j}\right\|_{H^{1}(\Omega)} \leq c_{3}
$$

According to $7,21,30$, the sequence $\left\{w_{j}\right\}$ is uniformly bounded in $C^{1, \alpha}(\bar{\Omega})$ for some $\alpha \in(0,1)$, and thus, for any $\beta \in(0, \alpha)$, the sequence $\left\{w_{j}\right\}$ has a subsequence $\left\{w_{j_{k}}\right\}$, denoted by $\left\{w_{j}\right\}$ that converges in $C^{1, \beta}(\bar{\Omega})$. Let us denote its limit as $w$.

We establish that $w>0$ on $\Omega$. It should be noted that $p:=\frac{2 N r}{N-2}>1$ as $1<q<\frac{N+2}{N-2}$. Then, according to $\left(\mathrm{f}_{1}\right)$ and the Sobolev embedding theorem, we obtain

$$
\begin{aligned}
\int_{\Omega}\left|f\left(u_{\lambda_{j}}\right)\right|^{p}\left\|u_{\lambda_{j}}\right\|_{\infty}^{-p} d x & \leq 2^{p-1} B^{p} \int_{\Omega}\left(u_{\lambda_{j}}^{p(q-1)}+1\right) d x \\
& \leq C\left(\left\|u_{\lambda_{j}}\right\|_{H^{1}(\Omega)}^{\frac{2 N}{N-2}}+1\right),
\end{aligned}
$$

where $C>0$ is a constant independent of $j$ and, without the loss of generality, we assume that $\left\|u_{\lambda_{j}}\right\|_{\infty}>1$. Thus, $\left\{\lambda_{j} f\left(u_{\lambda_{j}}\right)\left\|u_{\lambda_{j}}\right\|_{\infty}\right\}$ is bounded in $L^{p}(\Omega)$, which implies that it 
converges weakly in $L^{p}(\Omega)$. Let $z \in L^{p}(\Omega)$ be its weak limit. Since $\lambda_{j}\left\|u_{\lambda_{j}}\right\|_{\infty}^{-1} \rightarrow 0$ as $j \rightarrow \infty$ and $f\left(u_{\lambda_{j}}\right)$ is bounded from below, $z \geq 0$. Now, as

$$
\int_{\partial \Omega}\left|g\left(u_{\lambda_{j}}\right)\right|^{2} d \sigma \leq M_{g}^{2}|\partial \Omega|
$$

there exists a subsequence $\left\{g\left(u_{\lambda_{j_{k}}}\right)\right\}$ of $\left\{g\left(u_{\lambda_{j}}\right)\right\}$, denoted by $\left\{g\left(u_{\lambda_{j}}\right)\right\}$, and $\widetilde{g} \in L^{2}(\partial \Omega)$ such that $\left\{g\left(u_{\lambda_{j_{k}}}\right)\right\}$ converges weakly to $\widetilde{g}$ in $L^{2}(\partial \Omega)$. Evidently, $m_{g} \leq \widetilde{g} \leq M_{g}$ a.e. on $\partial \Omega$. Indeed, as $0<m_{g} \leq g\left(u_{\lambda_{j}}\right) \leq M_{g}<\infty, \forall j$, it holds that

$$
\int_{\partial \Omega} m_{g} \psi d \sigma \leq \int_{\partial \Omega} \tilde{g} \psi d \sigma \leq \int_{\partial \Omega} M_{g} \psi d \sigma, \quad \forall \psi \geq 0 \text { and } \psi \in L^{2}(\partial \Omega),
$$

which implies that $m_{g} \leq \widetilde{g} \leq M_{g}$ a.e. on $\partial \Omega$.

For $\psi \in C^{\infty}(\Omega)$, it holds that

$$
\begin{aligned}
\int_{\Omega} z \psi d x & =\lim _{j \rightarrow \infty} \int_{\Omega} \lambda_{j} f\left(u_{\lambda_{j}}\right)\left\|u_{\lambda_{j}}\right\|_{\infty}^{-1} \psi d x \\
& =\lim _{j \rightarrow \infty}\left(\int_{\Omega}\left(\nabla w_{j} \cdot \nabla \psi+V(x) w_{j} \psi\right) d x+\int_{\partial \Omega} g\left(u_{\lambda_{j}}\right) w_{j} \psi d \sigma\right) \\
& =\int_{\Omega}(\nabla w \cdot \nabla \psi+V(x) w \psi) d x+\int_{\partial \Omega} \widetilde{g} w \psi d \sigma .
\end{aligned}
$$

Thus, $-\Delta w+V(x) w=z \geq 0$ in $\Omega$, which implies that $\min _{\bar{\Omega}} w=\min _{\partial \Omega} w$ according to the weak maximum principle. If $\min _{\partial \Omega} w \geq 0$, then the claim is proven. Suppose that there exists $x_{0} \in \partial \Omega$ such that $\min _{\partial \Omega} w=w\left(x_{0}\right)<0$. As $w$ satisfies $\frac{\partial w}{\partial \eta}+\widetilde{g} w=0$ on $\partial \Omega$ and $\tilde{g}>0$, it can be seen that $\frac{\partial w}{\partial \eta}\left(x_{0}\right)>0$. This implies that there exists a local interior negative minimum. Let $x_{1}$ be its minimum point in $\Omega$. Then, $w\left(x_{1}\right)<0$ and $D_{i, j} w\left(x_{1}\right) \geq 0$, for all $i, j=1, \ldots N$, which contradicts with $-\Delta w+V(x) w \geq 0$ in $\Omega$. Thus, $w \geq 0$ on $\bar{\Omega}$. By applying the Hopf maximum principle, $\frac{\partial w}{\partial \eta}(x)<0$ for all $x \in \partial \Omega$, which gives $w>0$ on $\bar{\Omega}$.

Here, due to the convergence of $\left\{w_{j}\right\}$ to $w$ in $C^{1, \beta}$, for a sufficiently large $j, w_{j}(x)>0$ for all $x \in \Omega$. Therefore, $u_{\lambda_{j}}(x)>0$ for all $x \in \Omega$, which contradicts the assumption on the measure $m\left\{x \in \Omega: u_{\lambda_{j}}(x) \leq 0\right\}>0$. This proves Theorem 1.3 .

\section{Acknowledgments}

Ko was supported by a National Research Foundation of Korea (NRF) grant funded by the Korean Government (NRF-2017R1D1A1B03030681) and Sim was supported by a National Research Foundation of Korea (NRF) grant funded by the Korean Government (NRF2018R1D1A3A03000678). 


\section{References}

[1] H. Amann and M. G. Crandall, On some existence theorems for semi-linear elliptic equations, Indiana Univ. Math. J. 27 (1978), no. 5, 779-790.

[2] A. Ambrosetti, D. Arcoya and B. Buffoni, Positive solutions for some semi-positone problems via bifurcation theory, Differential Integral Equations 7 (1994), no. 3-4, 655663.

[3] T. Ando, A. B. Fowler and F. Stern, Electronic properties of two-dimensional systems, Rev. Modern Phys. 54 (1982), no. 2, 437-672.

[4] V. Anuradha, D. D. Hai and R. Shivaji, Existence results for superlinear semipositone BVP's, Proc. Amer. Math. Soc. 124 (1996), no. 3, 757-763.

[5] T. Bartsch and Z.-Q. Wang, Sign changing solutions of nonlinear Schrödinger equations, Topol. Methods Nonlinear Anal. 13 (1999), no. 2, 191-198.

[6] R. Bialecki and A. J. Nowak, Boundary value problems in heat conduction with nonlinear material and nonlinear boundary conditions, Appl. Math. Model. 5 (1981), no. $6,417-421$.

[7] H. Brezis and L. Nirenberg, $H^{1}$ versus $C^{1}$ local minimizers, C. R. Acad. Sci. Paris Sér. I Math. 317 (1993), no. 5, 465-472.

[8] D. Butler, E. Ko, E. Lee and R. Shivaji, Positive radial solutions for elliptic equations on exterior domains with nonlinear boundary conditions, Commun. Pure Appl. Anal. 13 (2014), no. 6, 2713-2731.

[9] A. Castro, D. G. de Figueredo and E. Lopera, Existence of positive solutions for a semipositone p-Laplacian problem, Proc. Roy. Soc. Edinburgh Sect. A 146 (2016), no. $3,475-482$.

[10] A. Castro, S. Gadam and R. Shivaji, Positive solution curves of semipositone problems with concave nonlinearities, Proc. Roy. Soc. Edinburgh Sect. A 127 (1997), no. 5, 921-934.

[11] P. Cherrier, Meilleures constantes dans des inégalités relatives aux espaces de Sobolev, Bull. Sci. Math. (2) 108 (1984), no. 3, 225-262.

[12] _ Problèmes de Neumann non linéaires sur les variétés riemanniennes, J. Funct. Anal. 57 (1984), no. 2, 154-206. 
[13] M. Chhetri, P. Drábek and R. Shivaji, Existence of positive solutions for a class of p-Laplacian superlinear semipositone problems, Proc. Roy. Soc. Edinburgh Sect. A 145 (2015), no. 5, 925-936.

[14] M. Cuesta and H. Ramos Quoirin, A weighted eigenvalue problem for the p-Laplacian plus a potential, NoDEA Nonlinear Differential Equations Appl. 16 (2009), no. 4, 469-491.

[15] J. M. Cushing, Nonlinear Steklov problems on the unit circle II (and a hydrodynamical application), J. Math. Anal. Appl. 39 (1972), 267-278.

[16] E. N. Dancer and J. Shi, Uniqueness and nonexistence of positive solutions to semipositone problems, Bull. London Math. Soc. 38 (2006), no. 6, 1033-1044.

[17] L. C. Evans, Partial Differential Equations, Second edition, Graduate Studies in Mathematics 19, American Mathematical Society, Providence, RI, 2010.

[18] D. Fasino and G. Inglese, Recovering unknown terms in a nonlinear boundary condition for Laplace's equation, IMA J. Appl. Math. 71 (2006), no. 6, 832-852.

[19] G. M. Figueiredo, J. R. Santos Júnior and A. Suárez, Structure of the set of positive solutions of a non-linear Schrödinger equation, Israel J. Math. 227 (2018), no. 1, $485-505$.

[20] J. Fleckinger, J. Hernández and F. de Thélin, Existence of multiple principal eigenvalues for some indefinite linear eigenvalue problems, Boll. Unione Mat. Ital. Sez. B Artic. Ric. Mat. (8) 7 (2004), no. 1, 159-188.

[21] J. Giacomoni, S. Prashanth and K. Sreenadh, $W^{1, N}$ versus $C^{1}$ local minimizers for elliptic functionals with critical growth in $\mathbb{R}^{N}$, C. R. Math. Acad. Sci. Paris 347 (2009), no. 5-6, 255-260.

[22] D. Gilbarg and N. S. Trudinger, Elliptic Partial Differential Equations of Second Order, Classics in Mathematics, Springer-Verlag, Berlin, 2001.

[23] P. V. Gordon, E. Ko and R. Shivaji, Multiplicity and uniqueness of positive solutions for elliptic equations with nonlinear boundary conditions arising in a theory of thermal explosion, Nonlinear Anal. Real World Appl. 15 (2014), 51-57.

[24] Y. Guo, Z.-Q. Wang, X. Zeng and H.-S. Zhou, Properties of ground states of attractive Gross-Pitaevskii equations with multi-well potentials, Nonlinearity 31 (2018), no. 3, 957-979. 
[25] F. Inkmann, Existence and multiplicity theorems for semilinear elliptic equations with nonlinear boundary conditions, Indiana Univ. Math. J. 31 (1982), no. 2, 213-221.

[26] O. Kavian and M. Vogelius, On the existence and "blow-up" of solutions to a twodimensional nonlinear boundary-value problem arising in corrosion modelling, Proc. Roy. Soc. Edinburgh Sect. A 133 (2003), no. 1, 119-149.

[27] E. Ko and S. Prashanth, Positive solutions for elliptic equations in two dimensions arising in a theory of thermal explosion, Taiwanese J. Math. 19 (2015), no. 6, 17591775.

[28] A. Le, Z.-Q. Wang and J. Zhou, Finding multiple solutions to elliptic PDE with nonlinear boundary conditions, J. Sci. Comput. 56 (2013), no. 3, 591-615.

[29] Y. Y. Li and M. Zhu, Sharp Sobolev inequalities involving boundary terms, Geom. Funct. Anal. 8 (1998), no. 1, 59-87.

[30] G. M. Lieberman, Boundary regularity for solutions of degenerate elliptic equations, Nonlinear Anal. 12 (1988), no. 11, 1203-1219.

[31] P.-L. Lions, On the existence of positive solutions of semilinear elliptic equations, SIAM Rev. 24 (1982), no. 4, 441-467.

[32] J.-q. Liu, Y.-q. Wang and Z.-Q. Wang, Solutions for quasilinear Schrödinger equations via the Nehari method, Comm. Partial Differential Equations 29 (2004), no. 5-6, 879901.

[33] J. López-Gómez, The maximum principle and the existence of principal eigenvalues for some linear weighted boundary value problems, J. Differential Equations 127 (1996), no. 1, 263-294.

[34] K. Medville and M. S. Vogelius, Existence and blow up of solutions to certain classes of two-dimensional nonlinear Neumann problems, Ann. Inst. H. Poincaré Anal. Non Linéaire 23 (2006), no. 4, 499-538.

[35] K. Perera, R. Shivaji and I. Sim, A class of semipositone p-Laplacian problems with a critical growth reaction term, Adv. Nonlinear Anal. 9 (2020), no. 1, 516-525.

[36] P. H. Rabinowitz, On a class of nonlinear Schrödinger equations, Z. Angew. Math. Phys. 43 (1992), no. 2, 270-291.

[37] M. Vogelius and J.-M. Xu, A nonlinear elliptic boundary value problem related to corrosion modeling, Quart. Appl. Math. 56 (1998), no. 3, 479-505. 
Eunkyung Ko

Major in Mathematics, College of Natural Science, Keimyung University, Daegu 42601, South Korea

E-mail address: ekko@kmu.ac.kr

Eun Kyoung Lee

Department of Mathematics Education, Pusan National University, Busan 46241, South Korea

E-mail address: eklee@pusan.ac.kr

Inbo Sim

Department of Mathematics, University of Ulsan, Ulsan 44610, South Korea

E-mail address: ibsim@ulsan.ac.kr 